\title{
Article \\ Changes in Overall Survival over Time for Patients with de novo Metastatic Breast Cancer
}

\author{
Toshiaki Iwase ${ }^{1,2} \mathbb{D}$, Tushaar Vishal Shrimanker ${ }^{1,2}$, Ruben Rodriguez-Bautista ${ }^{1,2}$, Onur Sahin ${ }^{1,2} \mathbb{D}_{\text {, }}$ \\ Anjali James ${ }^{1,2}$, Jimin $W^{3}{ }^{3}$, Yu Shen ${ }^{3}$ and Naoto T. Ueno ${ }^{1,2, *(D)}$
}

1 Section of Translational Breast Cancer Research, Department of Breast Medical Oncology, The University of Texas MD Anderson Cancer Center, 1515 Holcombe Boulevard, Houston, TX 77030, USA; tiwase@mdanderson.org (T.I.); tushaar.shrimanker@greenwichhospital.org (T.V.S.); rrodriguez15@mdanderson.org (R.R.-B.); osahin1@mdanderson.org (O.S.); ajames2@mdanderson.org (A.J.)

2 Morgan Welch Inflammatory Breast Cancer Research Program and Clinic, The University of Texas MD Anderson Cancer Center, 1515 Holcombe Boulevard, Houston, TX 77030, USA

3 Department of Biostatistics, The University of Texas MD Anderson Cancer Center, 1515 Holcombe Boulevard, Houston, TX 77030, USA; jwu5@mdanderson.org (J.W.); yshen@mdanderson.org (Y.S.)

* Correspondence: nueno@mdanderson.org; Tel.: +1-713-792-8754; Fax: +1-888-375-2139

check for

updates

Citation: Iwase, T.; Shrimanker, T.V.; Rodriguez-Bautista, R.; Sahin, O.; James, A.; Wu, J.; Shen, Y.; Ueno, N.T. Changes in Overall Survival over Time for Patients with de novo Metastatic Breast Cancer. Cancers 2021, 13, 2650. https://doi.org/ 10.3390/cancers 13112650

Academic Editors: Tommaso Susini and Nicoletta Biglia

Received: 7 May 2021

Accepted: 25 May 2021

Published: 28 May 2021

Publisher's Note: MDPI stays neutral with regard to jurisdictional claims in published maps and institutional affiliations.

Copyright: (c) 2021 by the authors Licensee MDPI, Basel, Switzerland. This article is an open access article distributed under the terms and conditions of the Creative Commons Attribution (CC BY) license (https:/ / creativecommons.org/licenses/by/ $4.0 /)$.
Simple Summary: The present study aimed to clarify the change in survival for de novo metastatic breast cancer over time. We found that overall survival significantly improved over time for the estrogen-receptor-positive, HER2-positive subtype, and exhibited a tendency toward improvement over time for the estrogen-receptor-negative, HER2-positive subtype. These results underscored the contribution of HER2-targeted therapy to survival.

Abstract: The purpose of this study was to determine the change in overall survival (OS) for patients with de novo metastatic breast cancer $(\mathrm{dnMBC})$ over time. We conducted a retrospective cohort study with 1981 patients with dnMBC diagnosed between January 1995 and December 2017 at The University of Texas MD Anderson Cancer Center. OS was measured from the date of diagnosis of dnMBC. OS was compared between patients diagnosed during different time periods: 5 -year periods and periods defined according to when key agents were approved for clinical use. The median OS was 3.4 years. The 5- and 10-year OS rates improved over time across both types of time periods. A subgroup analysis showed that OS improved significantly over time for the estrogen-receptorpositive/HER2-positive (ER+/HER2+) subtype and exhibited a tendency toward improvement over time for the ER-negative (ER-)/HER2+ subtype. In addition, median OS was significantly longer in patients with non-inflammatory breast cancer $(p=0.02)$ and patients with ER+ disease, progesterone-receptor-positive disease, HER2+ disease, lower nuclear grade, locoregional therapy, and metastasis to a single organ (all $p<0.0001$ ). These findings showed that OS at 5 and 10 years after diagnosis in patients with dnMBC improved over time. The significant improvements in OS over time for the ER+/HER2+ subtype and the tendency toward improvement for the ER-/HER2+ subtype suggest the contribution of HER2-targeted therapy to survival.

Keywords: breast neoplasms; neoplasm metastasis; inflammatory breast neoplasms; survival analysis

\section{Introduction}

De novo metastatic breast cancer $(\mathrm{dnMBC})$ is breast cancer that manifests metastasis at the time of initial diagnosis; dnMBC is different from recurrent metastatic breast cancer (rMBC). In the US, dnMBC accounted for approximately $6 \%$ of all breast cancer cases between 2005 and 2011 [1].

A previous retrospective study comparing clinical characteristics between patients with dnMBC and rMBC showed that patients with dnMBC tended to have higher rates of hormone receptor (HR)-negative disease, bone metastasis, liver metastasis, and a lower 
rate of central nervous system metastasis [2]. In survival analysis of 643 patients with $\mathrm{dnMBC}$ and 2881 with rMBC, the dnMBC patients had longer median overall survival (OS) (39.2 months vs. 27.2 months) [3]. Plausible reasons for the better OS of patients with dnMBC include that (1) dnMBC is chemonaïve, and a lower percentage of patients with dnMBC than with rMBC had originally chemo-resistant disease, and (2) general clinical characteristics of dnMBC patients such as bone-only metastasis and oligo-metastasis are dormant.

Although dnMBC differs clinically from rMBC, the general treatment strategy for dn$\mathrm{MBC}$ follows the rMBC treatment algorithm. In $\mathrm{rMBC}$, the introduction of newly developed chemotherapy and targeted therapy has contributed to improvement in survival over time. For instance, the introduction of drugs targeting human epidermal growth factor receptor 2 (HER2) drastically improved the survival of patients with rMBC [4]. However, little is known about how advances in treatment have changed survival for patients with dnMBC. Moreover, the impact of biological features and locoregional interventions on survival in patients with dnMBC needs to be elucidated. Clarifying these unknowns will contribute to a better understanding of dnMBC's biology and guide future research directions.

We hypothesized that the OS of patients with dnMBC has improved over time along with the development in drug therapy. The primary objective of this study was to test our hypothesis. The secondary objective was to identify biological and clinical prognostic factors for dnMBC.

\section{Materials and Methods}

\subsection{Inclusion Criteria}

We conducted a retrospective cohort study with prospectively collected data designed to determine the change in OS over time for dnMBC. We defined dnMBC as breast cancer with histologically proven distant metastasis at the time of initial diagnosis. Using this definition of dnMBC, we retrospectively searched the Breast Cancer Electronic Medical Record Management System at The University of Texas MD Anderson Cancer Center for patients diagnosed with dnMBC from January 1995 through December 2017. This search identified 2737 potentially eligible patients with dnMBC (Figure 1). Of these patients, 576 patients were excluded because of insufficient pathological information, including data on estrogen receptor (ER) status, progesterone receptor (PR) status, HER2 status, and grade. An additional 180 patients were excluded because of lack of information about inflammatory breast cancer (IBC, $\mathrm{N}=148)$, locoregional therapy $(\mathrm{N}=30)$, or site of metastasis $(\mathrm{N}=2)$. In total, 1981 patients with dnMBC were eligible for analysis. The primary outcome was OS, which was defined as the time from the date of the initial diagnosis until the date of death from any cause. The patient's time to death was censored at the last follow-up if the patient was alive without an event occurrence at the last follow-up.

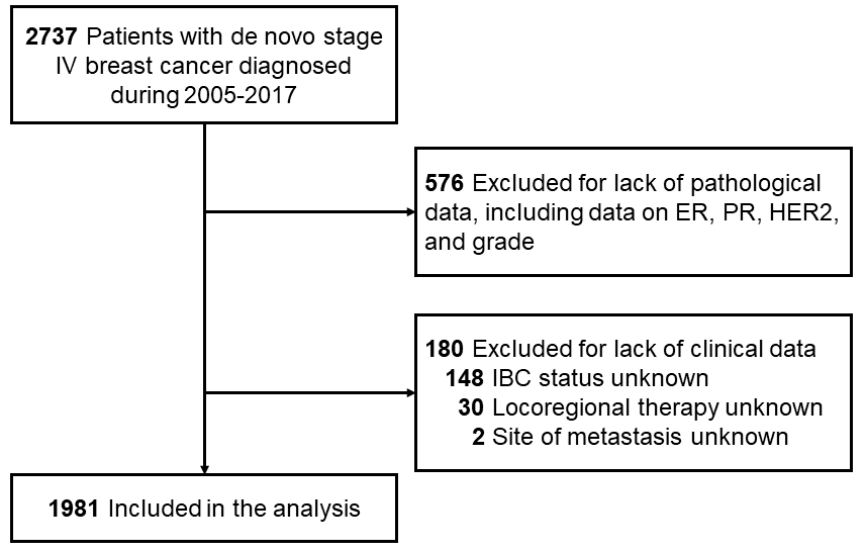

Figure 1. Patient selection process. 


\subsection{Definition of Time Periods}

To determine the changes in OS over time, two types of time periods were used: (1) time periods based on the date of approval of key agents by the U.S. Food and Drug Administration (FDA) and (2) 5-year periods. The key agents were trastuzumab, approved in 1999; fulvestrant, approved in 2002; ado-trastuzumab emtansine, approved in 2013; pertuzumab, approved in 2013. For triple-negative breast cancer (TNBC), PARP inhibitors were potential key drugs; however, we excluded PARP inhibitors because their potential to contribute to OS improvement seemed limited given the low prevalence of $B R C A 1$ and $B R C A 2$ mutations in previous reports [5-7].

\subsection{Definitions of Clinical and Pathological Variables}

Cases of dnMBC were defined as positive for ER or PR if at least $1 \%$ of cells expressed the hormone receptor by immunohistochemistry (IHC). HER2 positivity was determined according to the American Society of Clinical Oncology/College of American Pathologists guidelines at the time of pathological evaluation. IBC was a clinical diagnosis made by a multidisciplinary team consisting of a medical oncologist, surgical oncologist, radiologist, and nurse; IBC was diagnosed when patients exhibited one or more of a set of specific clinical manifestations, including the history of rapid onset of breast erythema, edema and/or peaud'orange, and/or warm breast, with or without an underlying palpable mass. A history of flattening, crusting, or retraction of the nipple was also considered. Locoregional treatment was defined as surgical resection of the primary tumor by any method and radiation therapy delivered to the area of the breast and regional lymph nodes regardless of the dose and the frequency of the treatment. Metastasis to a single organ was defined as metastasis in only one organ, such as bone, lung, brain, or soft tissue, including distant lymph nodes (but not regional lymph nodes), confirmed by imaging and pathology. Patients with multiple sites of metastasis in the same organ were considered to have metastasis to a single organ if that was the only organ involved.

\subsection{Statistical Considerations}

Data were summarized using standard descriptive statistics, such as mean, standard deviation, median, and range for continuous variables and frequency, and proportion for categorical variables. OS duration was estimated using the Kaplan-Meier method, and OS was compared between or among groups using the log-rank test. Univariate and multivariate Cox regression models were applied to assess the effect of variables of interest on OS. The model was selected by the backward method. We considered two-sided $p<0.05$ significant. All computations were carried out in SAS 9.4 (SAS Institute Inc., Cary, NC, USA). The study was approved by the Institutional Review Board of The University of Texas MD Anderson Cancer Center before data collection was initiated (protocol number: PA17-0613).

\section{Results}

\subsection{Patient Characteristics}

Table 1 shows the clinical characteristics of the patients included in the study. The median age was 52 years. Approximately 70\% of the patients had ER-positive (ER+) disease, and 28\% had HER2-positive (HER2+) disease. Fifteen percent of the patients had TNBC. Seventeen percent of the patients had IBC. Thirty-nine percent of the patients received locoregional therapy, and $62 \%$ had metastasis to a single organ. 
Table 1. Patient characteristics $(\mathrm{N}=1981)$.

\begin{tabular}{|c|c|c|}
\hline Characteristic & & Number of Patients (\%) \\
\hline \multirow{5}{*}{$\begin{array}{l}\text { Year of diagnosis, grouping } 1 \\
\text { (based on the date of approval } \\
\text { of key agents) }\end{array}$} & 1995-1998 & $72(4)$ \\
\hline & 1999-2003 & $305(15)$ \\
\hline & 2004-2008 & $469(24)$ \\
\hline & 2009-2012 & $564(29)$ \\
\hline & 2013-2017 & $571(29)$ \\
\hline \multirow{5}{*}{$\begin{array}{l}\text { Year of diagnosis, grouping } 2 \\
\text { (5-year periods) }\end{array}$} & 1995-1999 & $100(5)$ \\
\hline & $2000-2004$ & $353(18)$ \\
\hline & 2005-2009 & $517(26)$ \\
\hline & 2010-2014 & $676(34)$ \\
\hline & 2015-2017 & $335(17)$ \\
\hline \multicolumn{2}{|l|}{$\begin{array}{l}\text { Age at diagnosis, median } \\
\text { (range), year }\end{array}$} & $52(22-93)$ \\
\hline \multirow[t]{3}{*}{ ER status } & Positive & $1398(71)$ \\
\hline & Negative & $580(29)$ \\
\hline & Unknown & 3 \\
\hline \multirow[t]{3}{*}{ PR status } & Positive & $1051(53)$ \\
\hline & Negative & $920(47)$ \\
\hline & Unknown & 10 \\
\hline \multirow[t]{2}{*}{ HER2 status } & Positive & $555(28)$ \\
\hline & Negative & $1426(72)$ \\
\hline \multirow[t]{4}{*}{ Subtype } & ER+/HER2+ & $330(17)$ \\
\hline & ER+/HER2- & $1120(57)$ \\
\hline & ER $-/$ HER $2+$ & $225(11)$ \\
\hline & TNBC & $306(15)$ \\
\hline \multirow[t]{3}{*}{ Nuclear grade } & 1 & $96(5)$ \\
\hline & 2 & $754(38)$ \\
\hline & 3 & $1131(57)$ \\
\hline \multirow[t]{2}{*}{ IBC } & Yes & $339(17)$ \\
\hline & No & $1642(83)$ \\
\hline \multirow[t]{2}{*}{ Locoregional therapy } & Yes & $775(39)$ \\
\hline & No & $1206(61)$ \\
\hline \multirow[t]{2}{*}{ Metastasis to a single organ } & Yes & $1227(62)$ \\
\hline & No & $754(38)$ \\
\hline
\end{tabular}

\subsection{OS by Disease and Treatment Characteristics}

The median follow-up time was 7.4 years (95\% confidence interval [CI]: 6.99-8.22). A total of 1365 of the 1981 patients died by the end of the study period. The median OS interval was 3.4 years (95\% CI: 3.18-3.64) (Figure S1). The median OS interval was significantly longer in patients with non-IBC (vs. IBC) $(p=0.0210)$; median OS was also significantly longer in patients with ER+ disease (vs. ER-), PR+ disease (vs. PR negative [PR-]), HER2+ disease (vs. HER2-), lower nuclear grade, locoregional therapy (vs. none), and metastasis to a single organ (vs. multiple organs) (all $p<0.0001$ ) (Figure S2A-D,F-H). The Kaplan-Meier curves crossed at around 7 years for IBC (Figure S2A), 10 years for ER (Figure S2B), and 10 years for PR (Figure S2C). The ER+/HER2- subtype was associated with a better prognosis than the ER-/HER2+ subtype until 5 years after diagnosis when the curves crossed (Figure S2E). The ER-/HER2+ subtype and TNBC subtype survival curves plateaued after 5 years, but the survival curves of the ER+/HER2 - subtype and ER+/HER2+ subtype kept declining after 5 years (Figure S2E). 


\subsection{Prognostic Factors for OS}

Table 2 shows the univariate and multivariate Cox regression models with the primary outcome of OS. The risk was calculated as relative risk. The univariate analysis demonstrated that subtype, nuclear grade, locoregional therapy, and a number of organs with metastasis were significantly associated with OS. When other variables of interest were adjusted, the patients with ER+/HER2 - subtype had a 71\% higher risk of death than those with ER+/HER2+ subtype, and the patients with ER-/HER2+ subtype had a $49 \%$ higher risk of death than those with ER+/HER2+ subtype. Furthermore, the patients with TNBC had almost 4 times the risk of death in those with ER+/HER2+ disease. The patients with grade 3 disease had a $74 \%$ higher risk of death than those with grade 1 disease. The patients who received locoregional therapy had a $50 \%$ lower risk of death than those without locoregional therapy. The patients with metastasis to a single organ had a $36 \%$ lower risk of death than those with metastasis to multiple organs.

Table 2. Results of univariate and multivariate analysis for overall survival.

\begin{tabular}{|c|c|c|c|c|c|}
\hline Variables & & HR (95\% CI) & $p$-Value & HR (95\% CI) & $p$-Value \\
\hline \multirow[t]{4}{*}{$\begin{array}{l}\text { Year of diagnosis, } \\
\text { grouping } 1\end{array}$} & $\begin{array}{l}\text { 1995-1998 vs. } \\
2013-2017\end{array}$ & $1.12(0.85-1.48)$ & 0.4117 & & \\
\hline & $\begin{array}{c}1999-2003 \text { vs. } \\
2013-2017\end{array}$ & $1.11(0.92-1.32)$ & 0.2683 & & \\
\hline & $\begin{array}{l}2004-2008 \text { vs. } \\
2013-2017\end{array}$ & $1(0.85-1.18)$ & 0.9985 & & \\
\hline & $\begin{array}{l}\text { 2009-2012 vs. } \\
\text { 2013-2017 }\end{array}$ & $1.070(.91-1.26)$ & 0.3952 & & \\
\hline \multirow[t]{4}{*}{$\begin{array}{l}\text { Year of diagnosis, } \\
\text { grouping } 2\end{array}$} & $\begin{array}{l}1995-1999 \text { vs. } \\
2000-2004\end{array}$ & $0.91(0.72-1.15)$ & 0.4188 & & \\
\hline & $\begin{array}{l}2005-2009 \text { vs. } \\
2000-2004\end{array}$ & $0.8(0.69-0.93)$ & 0.0029 & & \\
\hline & $\begin{array}{l}\text { 2010-2014 vs. } \\
\text { 2000-2004 }\end{array}$ & $0.91(0.78-1.05)$ & 0.1857 & & \\
\hline & $\begin{array}{l}2015-2017 \text { vs. } \\
2000-2004\end{array}$ & $0.88(0.7-1.1)$ & 0.2541 & & \\
\hline Age at diagnosis & & 1.008 (1.004-1.013) & 0.0002 & & \\
\hline ER status & $\begin{array}{l}\text { Positive vs. } \\
\text { Negative }\end{array}$ & $0.59(0.53-0.59)$ & $<0.0001$ & & \\
\hline PR status & $\begin{array}{l}\text { Positive vs. } \\
\text { Negative }\end{array}$ & $0.64(0.57-0.64)$ & $<0.0001$ & & \\
\hline HER2 status & $\begin{array}{l}\text { Positive vs. } \\
\text { Negative }\end{array}$ & $0.64(0.56-0.64)$ & $<0.0001$ & & \\
\hline \multirow[t]{3}{*}{ Subtype } & $\begin{array}{c}\text { ER+/HER2- vs. } \\
\text { ER+/HER2+ }\end{array}$ & $1.54(1.31-1.54)$ & $<0.0001$ & $1.71(1.45-2.02)$ & $<0.0001$ \\
\hline & $\begin{array}{c}\text { ER - /HER2+ vs. } \\
\text { ER+/HER2+ }\end{array}$ & $1.41(1.13-1.41)$ & $<0.0001$ & $1.49(1.2-1.87)$ & 0.0004 \\
\hline & $\begin{array}{c}\text { TNBC vs. } \\
\text { ER+/HER2+ }\end{array}$ & 3.7 (3.05-3.7) & $<0.0001$ & $3.93(3.23-4.78)$ & $<0.0001$ \\
\hline \multirow[t]{2}{*}{ Nuclear grade } & 2 vs. 1 & 1.07 (0.82-1.07) & 0.0023 & $1.21(0.92-1.6)$ & 0.1677 \\
\hline & 3 vs. 1 & $1.51(1.16-1.51)$ & $<0.0001$ & $1.74(1.32-2.29)$ & $<0.0001$ \\
\hline IBC & Yes vs. No & $1.18(1.03-1.18)$ & 0.0212 & & \\
\hline $\begin{array}{c}\text { Locoregional } \\
\text { therapy }\end{array}$ & Yes vs. No & $0.53(0.47-0.53)$ & $<0.0001$ & $0.5(0.45-0.56)$ & $<0.0001$ \\
\hline $\begin{array}{l}\text { Metastasis to a } \\
\text { single organ }\end{array}$ & Yes vs. No & $0.59(0.53-0.59)$ & $<0.0001$ & $0.64(0.58-0.72)$ & $<0.0001$ \\
\hline
\end{tabular}




\subsection{Changes in OS over Time}

Figure 2 shows the OS rates for patients with dnMBC by diagnosis date, with dates grouped according to the timing of approval of key drugs (Figure 2A) and 5-year periods (Figure 2B). In both analyses, the 5-year and 10-year OS rates tended to improve over time, whereas the 1-year and 2-year OS rates did not. In the analysis based on the timing of approval of key drugs, the 5-year OS rate improved from 35\% (95\% CI: $24-46 \%$ ) for patients diagnosed in 1995-1998 to 38\% (95\% CI: 32-44\%) for those diagnosed in 2013-2017, and the 10-year OS rate improved from 8\% (95\% CI: 3-16\%) for patients diagnosed in 1995-1998 to $17 \%$ (95\% CI: $14-21 \%$ ) for those diagnosed in 2004-2008. In the analysis based on 5-year periods, the 5-year OS rate improved from 33\% (95\% CI: $24-42 \%$ ) for patients diagnosed in 1995-1999 to 43\% (95\% CI: 33-52\%) for those diagnosed in 2015-2017, and the 10-year OS rate improved from 11\% (95\% CI: 6-18\%) for patients diagnosed in 1995-1999 to $18 \%$ (95\% CI: $14-21 \%)$ for those diagnosed in 2005-2009.

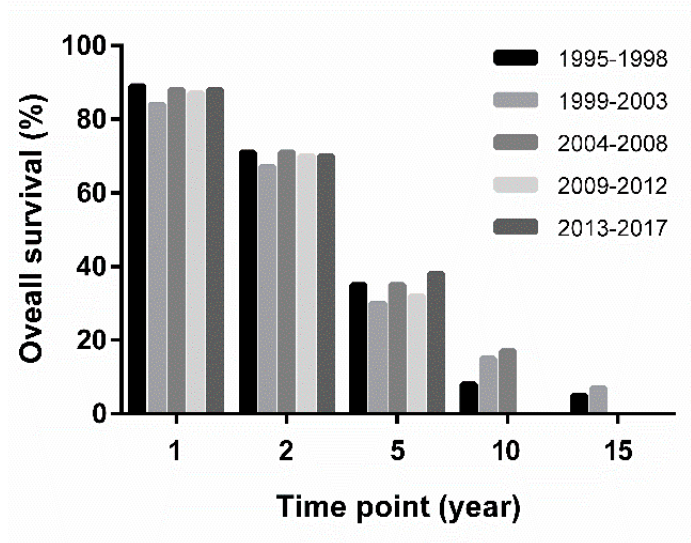

(A) The timing of approval of key drugs

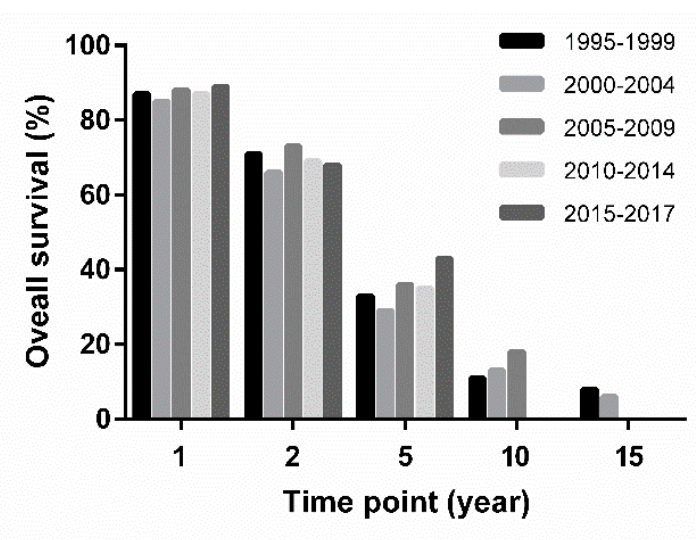

(B) 5-year periods

Figure 2. Overall survival rates for patients with de novo metastatic breast cancer at 1, 2, 5, 10, and 15 years after diagnosis according to diagnosis date, with dates grouped according to (A) the timing of approval of key drugs and (B) 5-year periods.

Next, we investigated the change in OS over time stratified by breast cancer subtype. We did not observe any significant change in OS over time for ER+/HER2- subtype or TNBC (Figure 3A,D). In contrast, OS for the ER+/HER2+ subtype significantly improved over time (Figure $3 \mathrm{~B}$ ). Although the result was not statistically significant, OS for the ER-/HER2+ subtype tended to improve over time (Figure 3C).

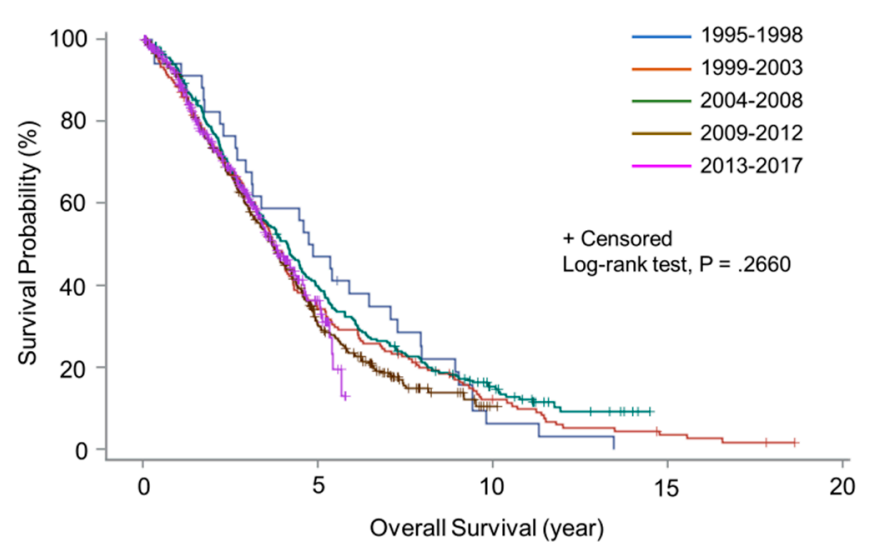

(A) ER+/HER2-

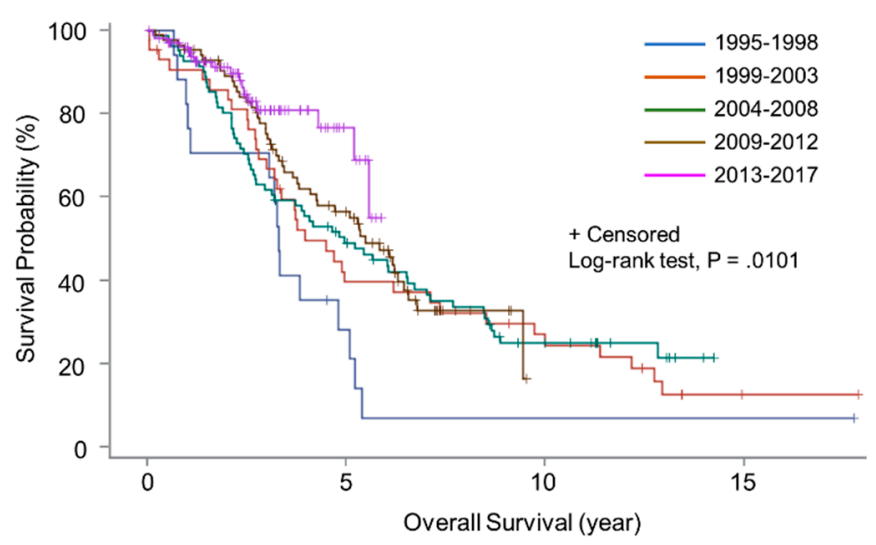

(B) ER+/ HER2+

Figure 3. Cont. 


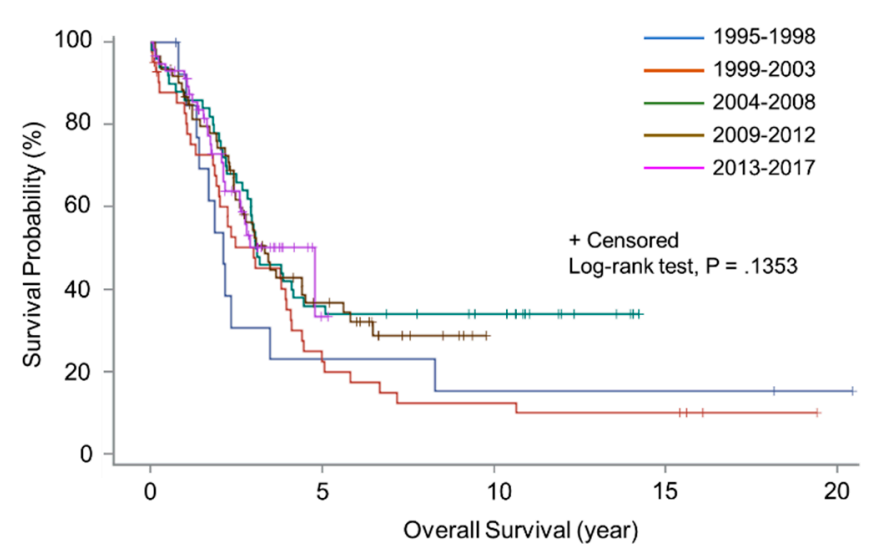

(C) ER-/HER2+

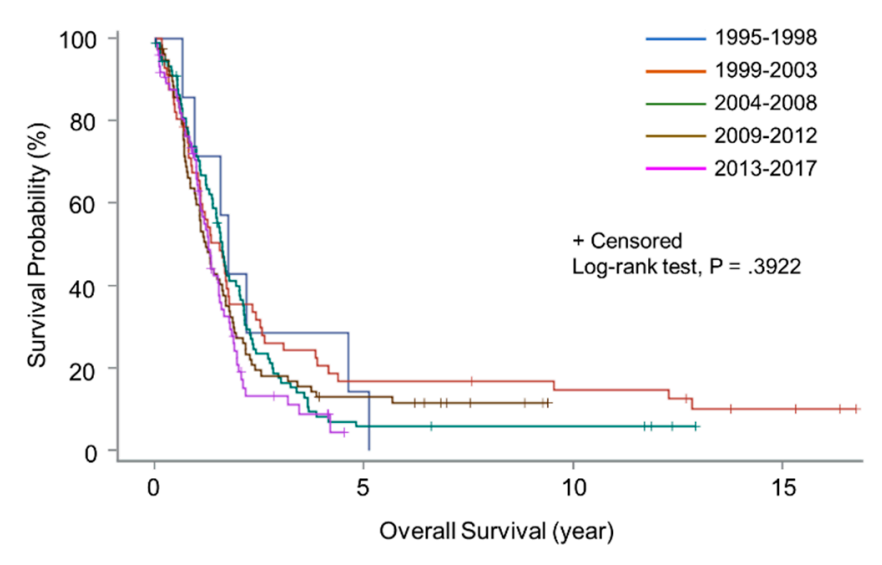

(D) TNBC

Figure 3. Overall survival curves for patients with de novo metastatic breast cancer according to year of diagnosis and breast cancer subtype: (A) estrogen receptor positive and HER2 negative (ER+/HER2-); (B) ER+/HER2 positive (HER2+); (C) ER-negative/HER2+; (D) triple-negative breast cancer.

\section{Discussion}

The present study showed that the 5-year and 10-year OS rates for patients with dnMBC tended to improve over time across time periods defined according to the dates of approval of key agents and across 5-year periods. In addition, OS improved significantly over time in the ER+/HER2+ subtype and tended to improve over time in the ER-/HER2+ subtype. Furthermore, we identified biological and clinical prognostic factors for dnMBC, including IBC status, ER status, PR status, HER2 status, subtype, nuclear grade, locoregional therapy, and a number of organs with metastasis.

Consistent with the results of a previous study of dnMBC versus rMBC [8], our study showed a tendency toward improvement in OS over time for dnMBC [8]; however, this was observed only 5 and 10 years after diagnosis. This result underscores the difficulty of improving the survival outcome for patients with dnMBC with progression within 2 years after diagnosis. In addition, our analysis revealed that many of the deaths within 2 years were in patients with TNBC, a subtype that did not show a significant improvement in OS over time.

TNBC does not have a specific druggable target. The PARP inhibitors could be a candidate for the treatment of TNBC; however, we estimated the contribution in terms of improving OS would be limited because the prevalence of $B R C A 1 / 2$ mutations in TNBC is approximately $10 \%$ to $20 \%$ in unselected cohorts [5-7]. A recent breakthrough in the treatment of metastatic TNBC is the use of an immune checkpoint inhibitor. The IMpassion130 trial showed that in patients with metastatic TNBC, PD-L1 blockade prolonged OS by approximately 4 months compared to OS in the control group [9]. Moreover, the subgroup of patients positive for PD-L1 by IHC demonstrated an improvement in OS of approximately 10 months with immune checkpoint inhibitor treatment. The survival benefit of immunochemotherapy with ICI is still under investigation in ongoing clinical trials, and the definitive results are expected to be available within the next 5 years. Given the current situation of drug development for metastatic TNBC, we expect that the novel treatment of dnMBC will contribute to the improvement of survival outcomes for TNBC in the next few years.

The subgroup analysis showed significant OS improvement for the ER+/HER2+ subtype and a tendency toward OS improvement for the ER-/HER2+ subtype over time. The OS improvement in HER2+ disease was due to HER2-targeted drugs, especially trastuzumab and pertuzumab. Those HER2-targeted drugs have revolutionized the treatment of HER2+ breast cancer and significantly prolonged survival. A systematic review of the efficacy of trastuzumab-containing regimens showed that they significantly decreased the HR for both death (HR, 0.82; 95\% CI, 0.71-0.94; $p=0.004)$ and disease progression (HR, 
$0.61 ; 95 \%$ CI, $0.54-0.70 ; p<0.00001$ ) [4]. Moreover, the combination of trastuzumab and pertuzumab with docetaxel prolonged OS by approximately 16 months compared with the placebo combination group [10]. In contrast to the efficacy of HER2-targeted drugs against HER2+ breast cancer, the efficacy of fulvestrant against ER+ and/or PR+ MBC was limited to improvements in disease progression; fulvestrant as monotherapy improved median progression-free survival by approximately 3 months [11]. However, the combination of fulvestrant with CDK 4/6 inhibitor showed a significant improvement of OS, with HRs of 0.746 and 0.71 , respectively, in the MONALEESA-2 and MONALEESA-7 trials [12,13]. Since the FDA approved CDK 4/6 inhibitor to treat metastatic HR+ breast cancer in 2015, we could not evaluate the survival effect of CDK $4 / 6$ inhibitor in the present study. An updated analysis after the next 5 years will be needed to evaluate the survival effect of CDK $4 / 6$ inhibitors in the real world.

As we expected, the clinical prognostic factors for dnMBC in the present studysubtype, nuclear grade, locoregional therapy, and number of organs with metastasis-were consistent with those previously reported [14,15]. Intriguingly, we observed that the survival curves for patients stratified by ER status and PR status crossed around 10 years. The survival curves of ER+ and PR+ groups kept declining and crossed the comparison group around 10 years after diagnosis. This result could be partly due to the acquired resistance to endocrine therapy during the long course of endocrine treatment. Once a tumor has acquired resistance, the selection of effective treatment becomes very difficult, and the survival outcome would be poor. In the present study, $\mathrm{HR}+\mathrm{dnMBC}$ had a better survival outcome than HER2+ dnMBC at baseline; however, overcoming resistance will be the key to further improve the survival outcome for the HR+ subgroup.

The multivariate analysis also showed that locoregional therapy and the number of organs with metastasis were prognostic factors. The efficacy of locoregional therapy for the primary tumor in patients with dnMBC has been discussed extensively. To date, three randomized clinical trials have been performed to answer the clinical question. A randomized controlled study from India with 716 patients with dnMBC found no survival benefit from early locoregional therapy [16]. In contrast, another randomized study by the Turkish Federation showed an improvement of OS by $17 \%$ with locoregional therapy [17]. The latest results of a large phase III randomized clinical trial with 256 patients with dnMBC (ECOG-ACRIN Research Group [E2108]) showed that locoregional therapy for the primary tumor did not significantly improve OS (3-year OS rate, $68.4 \%$ for the locoregional therapy group vs. $67.9 \%$ for the non-locoregional therapy group; $p=0.63$ by log-rank test) [18]. The major cause for those controversial results would come from a selection bias. It would be highly probable that the patient with limited disease, including oligo-metastasis, dormant tumor, and high sensitivity to systemic therapy may benefit from locoregional therapy. Although we did not count the number or measure tumor size in each metastatic site, $62 \%$ of the patients in our dataset had a single-organ metastasis. The amount of tumor burden and the site of metastasis would impact the effect of locoregional therapy on the survival outcome in the present study. We expect to obtain further insights from the Japan Clinical Oncology Group (JCOG) ongoing clinical trial, which has a similar design to E2108 [19]. Overall, the impact of locoregional therapy on the primary tumor on survival appears to be limited at this moment; however, the benefit of locoregional therapy for controlling local disease progression should be investigated to improve the patient's quality of life.

\section{Limitations}

The present study has several limitations. First, we excluded 756 of 2737 initially screened patients because of a lack of necessary pathological information. This process might have introduced a selection bias and affected the results. Second, we did not investigate the use of key drugs in each patient and did not evaluate the direct association between drug use and change in OS. Last, the methods of pathological evaluation, including the type of antibody, IHC procedure, and evaluation methods were not standardized 
throughout the study period. To overcome these limitations, a detailed evaluation via a well-designed prospective study would be crucial.

\section{Conclusions}

In this large retrospective cohort study, we confirmed that dnMBC tended to improve in OS over time, especially at the time points of 5 and 10 years after diagnosis. Although the improvement was not large, the significant OS improvement in the ER+/HER2+ subtype and the tendency toward OS improvement in the ER-/HER2+ subtype suggested the contribution of HER2-targeted therapy. The biological and clinical prognostic factors for dnMBC were similar to those described in previous reports, however, further investigation will be needed to elucidate the biology of dnMBC.

Supplementary Materials: The following are available online at https:/ / www.mdpi.com/article/10 .3390 / cancers13112650/s1, Figure S1. Kaplan-Meier curve of overall survival for all dnMBC patients. Figure S2. Kaplan-Meier curve of overall survival stratified by IBC (A), ER (B), PR (C), HER2 (D), subtypes $(E)$, grade $(F)$, the locoregional therapy $(G)$ and single or multiple organ metastasis $(H)$.

Author Contributions: Conceptualization, N.T.U., T.I.; methodology, N.T.U., T.I., J.W., Y.S.; software, J.W., Y.S.; validation, J.W., Y.S.; formal analysis, J.W., Y.S.; investigation, T.I., T.V.S., R.R.-B.; resources, T.I.; data curation, T.I., T.V.S., R.R.-B., O.S., A.J.; writing-original draft preparation, T.I.; writingreview and editing, Y.S., T.V.S., R.R.-B., N.T.U.; visualization, T.I., J.W., Y.S.; supervision, Y.S., N.T.U.; project administration, N.T.U.; funding acquisition, N.T.U. All authors have read and agreed to the published version of the manuscript.

Funding: The APC was funded by the Morgan Welch Inflammatory Breast Cancer Research Program, a State of Texas Rare and Aggressive Breast Cancer Research Program grant, National Institutes of Health/National Cancer Institute award 1R01CA205043-01A1 (to N.T.U.).

Institutional Review Board Statement: The study was conducted according to the guidelines of the Declaration of Helsinki and approved by the Institutional Review Board of The University of Texas MD Anderson Cancer Center before the data collection was initiated (protocol number: PA17-0613).

Informed Consent Statement: Patient consent was waived due to the retrospective study design.

Data Availability Statement: The data of the present study is not publicly available.

Acknowledgments: The authors thank Stephanie P. Deming for editing the manuscript (Research Medical Library, The University of Texas MD Anderson Cancer Center), Limin Hsu and Modesto G. Patangan, Jr, for data collection (Breast Medical Oncology, The University of Texas MD Anderson Cancer Center), and Huiming Sun for protocol development (Breast Medical Oncology, The University of Texas MD Anderson Cancer Center).

Conflicts of Interest: The authors declare no conflict of interest.

\section{References}

1. Howlader, N.; Krapcho, M. SEER Cancer Statistics Review, 1975-2012. Available online: http://seer.cancer.gov/csr/1975_2012/ (accessed on 1 November 2020).

2. Tripathy, D.; Brufsky, A.; Cobleigh, M.; Jahanzeb, M.; Kaufman, P.A.; Mason, G.; O'Shaughnessy, J.; Rugo, H.S.; Swain, S.M.; Yardley, D.A.; et al. De Novo Versus Recurrent HER2-Positive Metastatic Breast Cancer: Patient Characteristics, Treatment, and Survival from the SystHERs Registry. Oncologist 2020, 25, e214-e222. [CrossRef] [PubMed]

3. Dawood, S.; Broglio, K.; Ensor, J.; Hortobagyi, G.N.; Giordano, S.H. Survival differences among women with de novo stage IV and relapsed breast cancer. Ann. Oncol. 2010, 21, 2169-2174. [CrossRef] [PubMed]

4. Balduzzi, S.; Mantarro, S.; Guarneri, V.; Tagliabue, L.; Pistotti, V.; Moja, L.; D’Amico, R. Trastuzumab-containing regimens for metastatic breast cancer. Cochrane Database Syst. Rev. 2014, 2014, Cd006242. [CrossRef] [PubMed]

5. Gonzalez-Angulo, A.M.; Timms, K.M.; Liu, S.; Chen, H.; Litton, J.K.; Potter, J.; Lanchbury, J.S.; Stemke-Hale, K.; Hennessy, B.T.; Arun, B.K.; et al. Incidence and outcome of BRCA mutations in unselected patients with triple receptor-negative breast cancer. Clin. Cancer Res. 2011, 17, 1082-1089. [CrossRef]

6. Hartman, A.R.; Kaldate, R.R.; Sailer, L.M.; Painter, L.; Grier, C.E.; Endsley, R.R.; Griffin, M.; Hamilton, S.A.; Frye, C.A.; Silberman, M.A.; et al. Prevalence of BRCA mutations in an unselected population of triple-negative breast cancer. Cancer 2012, 118, 2787-2795. [CrossRef] 
7. Wong-Brown, M.W.; Meldrum, C.J.; Carpenter, J.E.; Clarke, C.L.; Narod, S.A.; Jakubowska, A.; Rudnicka, H.; Lubinski, J.; Scott, R.J. Prevalence of BRCA1 and BRCA2 germline mutations in patients with triple-negative breast cancer. Breast Cancer Res. Treat. 2015, 150, 71-80. [CrossRef] [PubMed]

8. Malmgren, J.A.; Mayer, M.; Atwood, M.K.; Kaplan, H.G. Differential presentation and survival of de novo and recurrent metastatic breast cancer over time: 1990-2010. Breast Cancer Res. Treat. 2018, 167, 579-590. [CrossRef] [PubMed]

9. Schmid, P.; Adams, S.; Rugo, H.S.; Schneeweiss, A.; Barrios, C.H.; Iwata, H.; Diéras, V.; Hegg, R.; Im, S.-A.; Wright, G.S.; et al. Atezolizumab and Nab-Paclitaxel in Advanced Triple-Negative Breast Cancer. N. Engl. J. Med. 2018, 379, 2108-2121. [CrossRef] [PubMed]

10. Swain, S.M.; Baselga, J.; Kim, S.-B.; Ro, J.; Semiglazov, V.; Campone, M.; Ciruelos, E.; Ferrero, J.-M.; Schneeweiss, A.; Heeson, S.; et al. Pertuzumab, Trastuzumab, and Docetaxel in HER2-Positive Metastatic Breast Cancer. N. Engl. J. Med. 2015, 372, 724-734. [CrossRef] [PubMed]

11. Robertson, J.F.R.; Bondarenko, I.M.; Trishkina, E.; Dvorkin, M.; Panasci, L.; Manikhas, A.; Shparyk, Y.; Cardona-Huerta, S.; Cheung, K.-L.; Philco-Salas, M.J.; et al. Fulvestrant $500 \mathrm{mg}$ versus anastrozole $1 \mathrm{mg}$ for hormone receptor-positive advanced breast cancer (FALCON): An international, randomised, double-blind, phase 3 trial. Lancet 2016, 388, 2997-3005. [CrossRef]

12. Hortobagyi, G.N.; Stemmer, S.M.; Burris, H.A.; Yap, Y.S.; Sonke, G.S.; Paluch-Shimon, S.; Campone, M.; Petrakova, K.; Blackwell, K.L.; Winer, E.P.; et al. Updated results from MONALEESA-2, a phase III trial of first-line ribociclib plus letrozole versus placebo plus letrozole in hormone receptor-positive, HER2-negative advanced breast cancer. Ann. Oncol. 2018, 29, 1541-1547. [CrossRef] [PubMed]

13. Im, S.-A.; Lu, Y.-S.; Bardia, A.; Harbeck, N.; Colleoni, M.; Franke, F.; Chow, L.; Sohn, J.; Lee, K.-S.; Campos-Gomez, S.; et al. Overall Survival with Ribociclib plus Endocrine Therapy in Breast Cancer. N. Engl. J. Med. 2019, 381, 307-316. [CrossRef] [PubMed]

14. Zeichner, S.B.; Herna, S.; Mani, A.; Ambros, T.; Montero, A.J.; Mahtani, R.L.; Ahn, E.R.; Vogel, C.L. Survival of patients with de-novo metastatic breast cancer: Analysis of data from a large breast cancer-specific private practice, a university-based cancer center and review of the literature. Breast Cancer Res. Treat. 2015, 153, 617-624. [CrossRef] [PubMed]

15. Lane, W.O.; Thomas, S.M.; Blitzblau, R.C.; Plichta, J.K.; Rosenberger, L.H.; Fayanju, O.M.; Hyslop, T.; Hwang, E.S.; Greenup, R.A. Surgical Resection of the Primary Tumor in Women With De Novo Stage IV Breast Cancer: Contemporary Practice Patterns and Survival Analysis. Ann. Surg. 2019, 269, 537-544. [CrossRef] [PubMed]

16. Badwe, R.; Hawaldar, R.; Nair, N.; Kaushik, R.; Parmar, V.; Siddique, S.; Budrukkar, A.; Mittra, I.; Gupta, S. Locoregional treatment versus no treatment of the primary tumour in metastatic breast cancer: An open-label randomised controlled trial. Lancet Oncol. 2015, 16, 1380-1388. [CrossRef]

17. Soran, A.; Ozmen, V.; Ozbas, S.; Karanlik, H.; Muslumanoglu, M.; Igci, A.; Canturk, Z.; Utkan, Z.; Ozaslan, C.; Evrensel, T.; et al. Randomized Trial Comparing Resection of Primary Tumor with No Surgery in Stage IV Breast Cancer at Presentation: Protocol MF07-01. Ann. Surg. Oncol. 2018, 25, 3141-3149. [CrossRef] [PubMed]

18. Khan, S.A.; Zhao, F.; Solin, L.J.; Goldstein, L.J.; Cella, D.; Basik, M.; Golshan, M.; Julian, T.B.; Pockaj, B.A.; Lee, C.A.; et al. A randomized phase III trial of systemic therapy plus early local therapy versus systemic therapy alone in women with de novo stage IV breast cancer: A trial of the ECOG-ACRIN Research Group (E2108). J. Clin. Oncol. 2020, 38, LBA2. [CrossRef]

19. Shien, T.; Mizutani, T.; Tanaka, K.; Kinoshita, T.; Hara, F.; Fujisawa, T.; Masuda, N.; Tamura, K.; Hojo, T.; Kanbayashi, C.; et al. A randomized controlled trial comparing primary tumor resection plus systemic therapy with systemic therapy alone in metastatic breast cancer (JCOG1017 PRIM-BC). J. Clin. Oncol. 2017, 35, TPS588. [CrossRef] 